J. of the Korean Sensors Society

Vol. 19 , No. 6 (2010) pp. $403-420$

DOI : $10.5369 /$ JSST.2010.19.6.403

pISSN 1225-5475/eISSN 2093-7563

\title{
Nano and micro structures for label-free detection of biomolecules
}

\author{
Kilho Eom, Taeyun Kwon*, and Young-Soo Sohn**,
}

\begin{abstract}
Nano and micro structure-based biosensors are promising tool for label-free detection of biomolecular interactions with great accuracy. This review gives a brief survey on nano and micro platforms to sense a variety of analytes such as DNA, proteins and viruses. Among incredible nano and micro structure for bio-analytical applications, the scope of this paper will be limited to micro and nano resonators and nanowire field-effect transistors. Nanomechanical motion of the resonators transducers biological information to readable signals. They are commonly combined with an optical, capacitive or piezo-resistive detection systems. Binding of target molecule to the modified surface of nanowire modulates the current of the nanowire through electrical field-effect. Both detection methods have advantages of label-free, real-time and high sensitive detection. These structures can be extended to fabricate array-type sensors for multiplexed detection and highthroughput analysis. The biosensors based on these structures will be applied to lab-on-a-chip platforms and point-ofcare diagnostics. Basic concepts including detection mechanisms and trends in their fields will be covered in this review.
\end{abstract}

Key Words : nano/micro structure, label-free, biosensors, resonator, nanowire FET

\section{Introduction}

It is of high importance to study interactions between biomolecules, to detect specific biomolecules from bodily fluid, and to observe the activity of cells, which are the main theme in life science research ${ }^{[1]}$, eventually resulting in the improvement of the health and welfare. For this reason, the field of the bio-sensing has become one of research areas that are currently received a lot of attention in scientific community as well as engineering community $^{[2]}$. The biosensors are generally composed of a biologically recognition element which is able to specifically capture the target analyte, and a physicochemical transducer that converts the biological recognition events into readable signals such as electrical or optical signals. The current trends is towards miniaturization of the sensor device since scaling down of a device leads to the large surface-to-volume ratio resulting in the increase of detection sensitivity. Key technology to fabricate miniaturized devices is to reduce the size of the transducers. A variety of sensor architectures

Department of Mechanical Engineering, Korea University * Department of Biomedical Engineering, Yonsei University

**Department of Biomedical Engineering, Catholic University of Daegu

Corresponding author: sohnys@cu.ac.kr

(Received: September 16, 2010, Revised: October 15, 2010,

Accepted : October 15, 2010) has been developed, e.g. cantilevers, nanowires, nanotubes, quantum dots, surface plasmon resonance, and nanogaps. Sensor architecture employs the physicochemical transducing element, which can be usually classified into two types; labeling-based and label-free transducing systems. The labeling system incorporates chemical labels such as fluorescence and radio active materials during detection. These methods are convenient and versatile. However, the labeling process can affect the characteristics of the target ananlytes. Moreover, the procedure is laborous and time-consuming. This has recently led many research groups to develop the de novo sensing toolkit enabling the label-free detection with high sensitivity that can be achieved through miniaturization $^{[3]}$.

In this review, we will focus on the miniaturized biosensors based on nano and micro structures. Our review is restricted to resonators and nanowire fieldeffect transistors, which are the representative labelfree nano and micro sensors. Our review describes the fundamental principles for label-free detections using nanoresonators and nanowire field effect transistor, which will eventually provide the fundamental insights into not only the current state-of-art in label-free detections but also the novel sensing paradigms for future sensing technologies. 


\section{Label-Free Detection through Mechanical Transduction}

Over a last decade, nanomechanical detection principle has been considered with a lot of interests because this principle enables not only the sensitive detection even at atomic resolution ${ }^{[4-6]}$ but also the detection of various physical quantities such as surface stress ${ }^{[7]}$, surface elastic stiffness ${ }^{[8]}$, and so forth. Specifically, the fundamental for such detection scheme is the direct transduction of molecular binding between analytes (target molecules) and receptor molecules on the nanomechanical sensor surface into the mechanical deformation change(e.g. deflection change, and resonance shift) of the sensor. Here, we focus our review on the label-free detection using micro/nanocantilevers and/or nanostructures such as nanotubes based on their resonance motion.

\subsection{Detection Principles}

\subsubsection{Molecular Detection Using Bending Deflection} Motion

For a last decade, microcantilevers have received the significant attention because of their ability to sense not only the biological species but also the molecular interactions ${ }^{[9-11]}$. The label-free detection using microcantilevers has been conducted based on deflection-based measurements. Specifically, the molecular adsorptions of specific biological species onto the cantilever surface leads to the change of the surface state, and consequently the surface stress change that results in the bending deflection change of microcantilever(see Fig. 1). In particular, the surface stress change due to molecular adsorption can be measured from the bending deflection change through the Stoney's formula ${ }^{[12]}$.

$$
\Delta \tau=\frac{E}{3(1-v)}\left(\frac{t}{L}\right)^{2} \Delta \omega
$$

where $\Delta \tau$ is the surface stress change, $\Delta w$ is the bending deflection change of a cantilever, and $E, v, t$, and $L$ represent the elastic modulus, the Poisson's ratio, the thickness, and the length of a cantilever, respectively.

The label-free detection based on bending deflection motion of a cantilever was first utilized for studying the atomic adsorption onto the surface. In particular, the atomic adsorption onto the cantilever surface was observed by measuring the bending deflection change

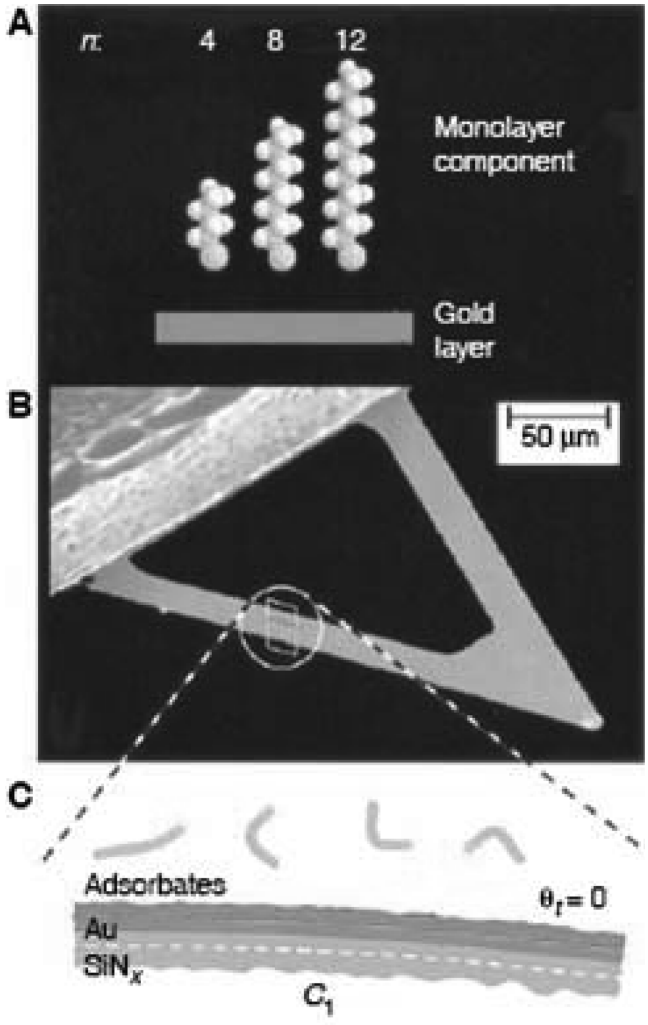

D

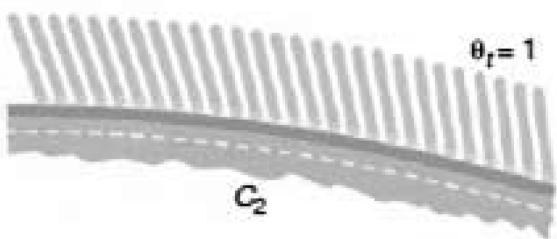

Fig. 1. Schematic illustration of atomic adsorption-induced cantilever bending motion. When alkane thiol groups are adsorbed onto a cantilever's surface, the intermolecular interactions between such adsorbed alkane thiols generate the surface stress change that leads to the cantilever bending deflection change. From $^{[13]}$. Reprinted with permission from AAAS (copyright 1997 the american association for the advancement of science).

due to such adsorption. The surface stress change due to atomic adsorption onto the surface was estimated from the bending deflection change based on Stoney's formula as described in Eq. (1). Gerber and coworkers ${ }^{[13]}$ found that the surface stress change due to atomic adsorption is well depicted by Langmuir kinetic model, and also that the role of molecular configurations on the 
surface stress change is also quantitatively described by the surface stress change. This indicates that microcantilever-based detection based on deflection motion may be a viable tool that enables the fundamental insights into the molecular adsorption.

Moreover, the bending deflection-based sensing was recently employed for the label-free detection of specific biological species related to diseases for further potential applications to early diagnostics. For such label-free detection, the chemical modification of a sensing surface is a priori requisite such that a specific receptor molecules are immobilized on the surface as follows: (i) immobilization using chemical reaction between amine group of molecules and silicon surface ${ }^{[14]}$, (ii) functionalization through chemical conjugation between thiol group of molecules and gold surface ${ }^{[15]}$, and (iii) immobilization based on self-assembled monolayer that is able to be chemically conjugated to carboxyl group of molecules ${ }^{[16]}$. Based on a cantilever functionalized with specific receptor molecules based on surface chemistry as described ${ }^{[17]}$, recent studies ${ }^{[18-22]}$ reported the sensitive label-free detection of specific biological species such as antigen ${ }^{[18]}, \mathrm{RNA}^{[19]}, \mathrm{DNA}^{[20-22]}$, and so forth. Recently, it has been notably reported that bending deflection-based cantilever assay allows the quantitative understanding of molecular interactions between peptides related to diseases and drug molecules and/or drug-resistance of mutated peptide molecules ${ }^{[23]}$. This highlights the potential of cantilever-based bioassay for sensitive detection of biomarkers and/or drug-screening.

Despite its capability of sensitive detection, the bending deflection-based cantilever bioassay exhibits a couple of restrictions as follows: (i) As described in Eq. (1), if cantilever length is too small, the bending deflection change due to surface stress change originated from molecular binding is difficult to be measurable using optical apparatus. This prevents a cantilever from being scaled down from micro-scales to nano-scales. (ii) In spite of straightforward detection principle, the bending deflection-based bioassay does not allow one to quantify the amount of target molecules that are involved in the molecular interaction.

\subsubsection{Resonance-Based Molecular Detection}

In recent years, a novel detection scheme has been introduced in such a way that the resonant frequency behaviors of micro/nanoscale devices are measured in response to molecular binding on the surface of a device ${ }^{[17,24]}$. That is, if specific molecules are selectively bound to the surface of a device, then molecular adsorption leads to the increase of the effective mass of a device, which results in the decrease of the resonant frequency. In general, the resonant frequency shift due to molecular binding is linearly proportional to the total mass of chemisorbed molecules ${ }^{[24,25]}$. In the context of linear elasticity, the resonant frequency shift due to molecular chemisorption is represented in the form of ${ }^{[24,25]}$

$$
\frac{\Delta \omega}{\omega_{0}}=-\frac{1}{2} \frac{\Delta m}{M}
$$

where $\Delta \omega$ is the resonant frequency shift, $\omega$ is the resonant frequency of a bare device(without any molecular adsorption), $\Delta m$ is the total mass of chemisorbed molecules, and $M$ is the effective mass of a bare device. Here, a negative sign indicates the decrease in the resonant frequency in case of molecular adsorption. Even though the detection principle described in Eq. (2) is straightforward, the resonance-based sensing based on small-scale device is quite complicated due to unexpected effects arising from nanoscale physics. For instance, if a device is scaled down to nano-scales, the surface effect such as surface stress and/or surface elastic stiffness plays a critical role in the mechanical motion of such a nanoscale device ${ }^{[8,26-28]}$. In particular, the scaling down of a device causes the creation of a surface resulting in the increase of surface energy $E_{S}$ that is generally dependent on the mechanical strain ${ }^{[28]}$. The surface stress is then defined as $\tau=\partial E_{s} / \partial \varepsilon$, where $\varepsilon$ is the mechanical strain. This indicates that the detection principle using nanoscale resonators has to include not only the mass effect but also the surface effect. This surface effect will be demonstrated later in Section 2.3.2.

The resonance-based detection has recently been received much attention in the scientific community, because scaling down of a resonator allows the ultrahigh sensitive detection of molecules even at atomic resolution. For instance, Roukes' group ${ }^{[4]}$ at Caltech first reported the ultrahigh sensitive detection of atomic mass in zepto-gram $\left(10^{-21} \mathrm{~g}\right)$ resolution using nanoresonators(see Fig. 2). In a similar spirit, Zettl's group ${ }^{[6]}$ at UC Berkeley showed that carbon nanotube(CNT) resonator is able to sense and detect gold atoms at atomic resolution because of its high resonant frequency in the 


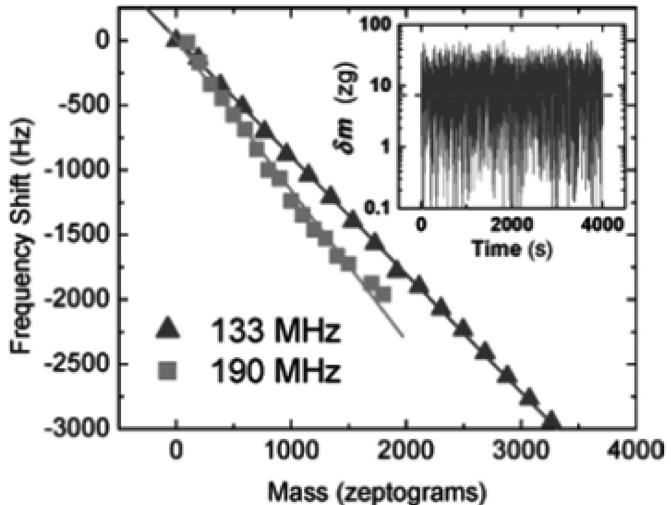

Fig. 2. Resonant frequency change due to atomic adsorption for NEMS resonators, which exhibit the resonant frequencies of $133 \mathrm{MHz}$ and $190 \mathrm{MHz}$, respectively. The resonant frequencies were measured in cryogenic, ultrahigh-vacuum conditions. The minimum detectable mass for NEMS resonators is $\sim 7 \mathrm{zg}$, which is equivalent to atomic masses of $\sim 30 \mathrm{Xe}$ atoms. Reprinted with permission from $^{[4]}$. Copyright 2006 American Chemical Society.

$\mathrm{GHz}\left(10^{9} \mathrm{~Hz}\right)$ regime. Even though nanoscale resonator enables the sensitive detection even at atomic resolution, as stated above, the detection principle demonstrated in Eq. (2) may be insufficient to describe the dynamic behavior of nanoresonators in response to molecular adsorption due to unexpected effects such as surface effects.

\subsection{Resonator-Based Molecular Recognitions}

As described earlier, resonator-based sensing allows not only the sensitive detection but also the quantification of molecular binding ${ }^{[14,29]}$. For example, nanomechanical resonators enable the measurement of molecular weight of specific molecules and/or cells ${ }^{[30-33]}$ for further characterization and/or quantification of their biological functions. Despite numerous recent studies on resonator-based detection, this section will provide representative epitomes of label-free detection using resonators.

In early 2000 s, resonant microcantilevers were taken into account for sensing and detecting the specific cells and/or virus. For instance, Craighead group ${ }^{[34]}$ at Cornell reported the cell detection using resonant microcantilevers by measuring the resonant frequency shift due to cell adsorption onto a cantilever surface. They

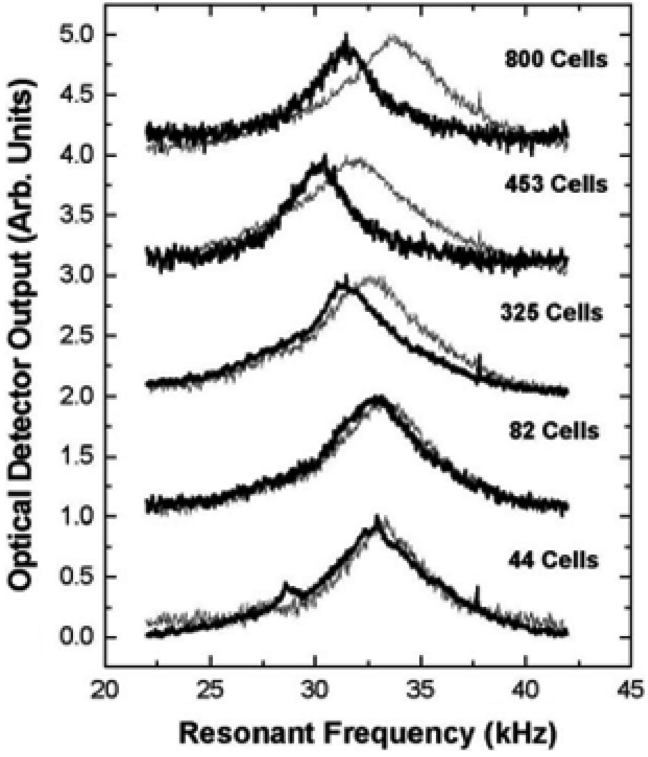

Fig. 3. Resonant frequency-behavior of a microcantilever in response to cell adsorption. Cellular adsorption onto a cantilever leads to the increase of effective mass of a cantilever, which results in the decrease of resonant frequency. The minimum detectable mass using resonant microcantilever is $\sim 6 \mathrm{pg}$, which is equivalent to the total mass of $\sim 15$ E.colicells. Reprinted with permission from ${ }^{[34]}$. Copyright 2000 American Institute of Physics.

estimated the mass of adsorbed cells based on the detection principle described in Eq. (2) [see Fig. 3]. In a similar spirit, Bashir's group ${ }^{[35]}$ at UIUC provided the labelfree detection of cells using cantilevers based on the measurement of resonant frequency shift. It should be kept in mind that these cell detections reported in previous studies ${ }^{[34,35]}$ are based on the measurement of resonant frequencies of a cantilever operated in dry air rather than aqueous environment. This indicates that these studies ${ }^{[34,35]}$ are restricted to characterize the cell's function in a real time, because a cell performs the function in aqueous environment. In general, because of low quality factor(Q-factor) in aqueous environment ${ }^{[36]}$, resonators are still unfavorable for in situ detection of specific biological species in physiological conditions. Recently, Manalis group ${ }^{[30,31,37]}$ at MIT developed a novel resonant device, called as suspended microchannel resonator(SMR), that is, microchannel is embedded in a microcantilever. Their SMR is able to exhibit the high detection sensitivity due to the detection scheme 


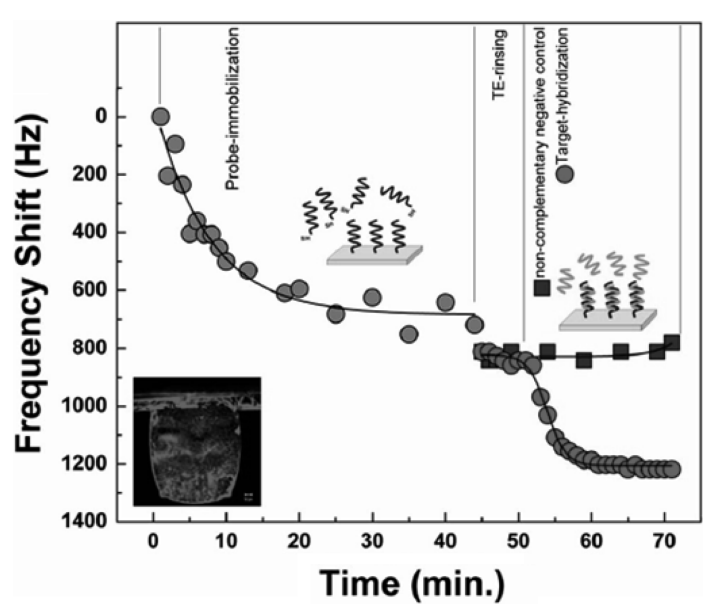

Fig. 4. The resonant frequency response for a microcantilever to DNA immobilization as well as DNA hybridization. The resonant frequency shift due to not only DNA immobilization but also DNA hybridization is well depicted by exponential curves, which implies that frequency shift due to DNA adsorption can be described by Langmuir kinetics. Reprinted with permission from ${ }^{[39]}$. Copyright 2008 American Institute of Physics.

such that specific biological species are detected inside a microchannel embedded in a cantilever while a cantilever vibrates in dry air with high Q-factor. They showed that SMR is able to weigh a single cell with high precision ${ }^{[30]}$. More remarkably, the characterization of cell growth during a cell cycle has been made using SMR. Specifically, based on measurement of resonant frequency shift, they are able to measure the mass increase rate for cell growth ${ }^{[31]}$.

In recent years, micro/nanomechanical resonators have been widely employed for sensing and detection of biological molecules such as DNA and/or proteins. DNA detection has been dated back to early 2000's in which Dravid group ${ }^{[38]}$ at Northwestern reported the resonant microcantilever-based DNA detection. In their work $^{[38]}$, for sensitive detection, they have used nanoparticles as mass amplification such that when target DNA is chemically conjugated to probe DNA(which is shorter than target DNA), a secondary complimentary DNA conjugated to nanoparticle is then injected so as to be conjugated to hybridized DNA between target DNA and probe DNA. In spite of sensitive detection, nanoparticle conjugation prevents them from measuring the mass of target molecules bound to a functionalized cantilever. Craighead group ${ }^{[29]}$ has recently reported a nanomechanical resonator and its great potential to sense and detect DNA even at single-molecule resolution with measurement of molecular weight of such DNA. Their detection was implemented by using a resonator vibrating in high-vacuum condition. Recently, Kwon et al. ${ }^{[39]}$ have recently suggested resonant microcantilever-based in situ, label-free detection of DNA chain(see Fig. 4). Specifically, Kwon et al. ${ }^{[39]}$ measured the resonant frequency shift for a microcantilever vibrating in aqueous environment due to DNA hybridization. Furthermore, it is shown that the resonant frequency shift due to DNA hybridization is well depicted by Langmuir kinetic model that allows the extraction of the kinetic rate of DNA hybridization.

Moreover, resonant devices have recently been utilized for protein detections. For example, Lee et al. ${ }^{[40]}$ considered microcantilever vibrating in dry air for sensing the specific proteins. Their detection resides in the measurement of shift in a resonant frequency due to loading of proteins onto a cantilever. They argued that the mass of chemisorbed proteins is insufficient to depict the resonant frequency shift due to protein adsorption, and that such resonant frequency shift is attributed to the surface stress originated from proteinprotein interactions ${ }^{[40]}$. This argument will be more delineated later in Section 2.3.2. Kwon et al. ${ }^{[16]}$ reported the in situ, label-free detection of prostate specific antigen(PSA) in a real-time using vibrant microcantilever immersed in buffer solution. Their detection is ascribed to relatively high Q-factor in liquid. Moreover, they showed that the resonant frequency shift measured in buffer solution due to protein chemisorption is contributed by not only the mass of adsorbed proteins but also the hydrophilicity change during protein chemisorptions $^{[16]}$. Recently, Craighead group ${ }^{[41]}$ provided the sensitive, label-free detection using resonant microcantilevers with nanoparticle-based amplifications. In particular, in order to amplify the resonant frequency shift due to target binding onto a cantilever, the nanoparticles conjugated to secondary antibody that is able to capture the target were introduced as an amplifier such that when nanoparticles conjugated to secondary antibody attach to the target molecules bound to a cantilever, the resonant frequency shift is amplified due to relatively large mass of nanoparticles. Based on this scheme, a detection of proteins in a concentration of $\sim \mathrm{fg} / \mathrm{ml}$ was achieved. 


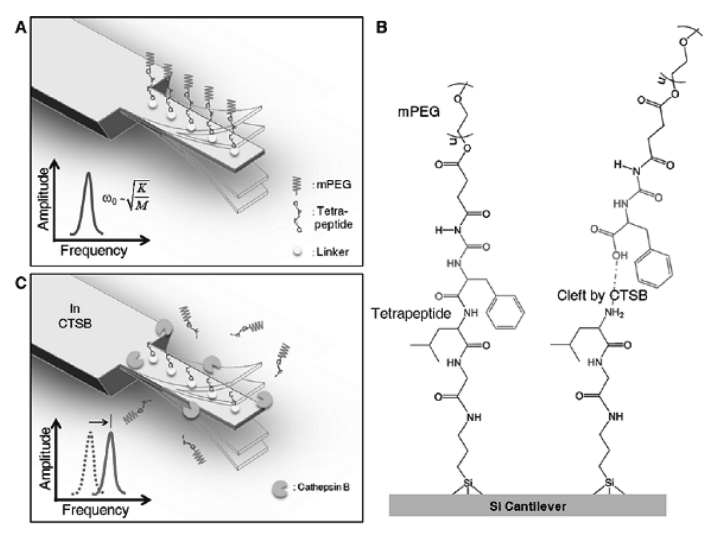

Fig. 5. Schematic illustration of resonator-based detection of proteolysis of peptides by protease. (A) A cantilever functionalized by a specific peptide sequence GFLG(GlyPheLysGly) conjugated to PEG chain vibrates with a resonant frequency of $w_{0}$. (B) Chemical structure of peptide chain GFLG conjugated to PEG chain is shown. A protease, cathepsin $\mathrm{B}(\mathrm{CTSB})$, is able to cleave the covalent bond between GF and LG resulting in the enzymatic cleavage of GFLG. (C) Enzymatic cleavage of GFLG chain immobilized on a cantilever leads to decrease in the effective mass, which results in the increase of resonant frequency. Figure is adopted from ${ }^{[14]}$.

Despite numerous experimental works on label-free detection of cells, DNA, and proteins, a resonant device has not been widely utilized for studying the drugenzyme-protein interactions which plays a critical role in the cellular signal transduction. Specifically, enzyme takes a dominant role for cleavage of specific proteins and/or peptide sequences so as to produce the substrate (that is cleft protein or peptide) which leads to cellular signaling cascade that determines the cell function ${ }^{[42,43]}$. It is thus of high importance to understand the proteinenzyme interactions and/or protein-enzyme-drug interactions for further insights into cellular signaling mechanism and/or regulation of such signaling. Recently, Kwon et al. ${ }^{[14]}$ have reported the label-free, in situ, monitoring of peptide-enzyme interactions using vibrant microcantilevers immersed in buffer solution(see Fig. 5). They have measured the enzyme concentration-dependent proteolysis efficacy based on the measurement of frequency shift due to enzymatic cleavage of peptides on the cantilever surface. Further, they have employed the Langmuir kinetic model that is applied to the meas- ured frequency shift due to enzymatic cleavage in a real-time. It is shown that Langmuir kinetic model is suitable to describe the enzymatic cleavage mechanism, and that the kinetics of enzymatic cleavage was obtained as a function of enzyme concentration. In addition, Kwon and coworkers ${ }^{[14]}$ have recently studied the inhibition mechanism of proteolysis using resonant microcantilevers operated in buffer solution. These studies shed light on the great potential of resonant devices for studying the enzymatic activity and/or its inhibition that may provide a key concept of how to regulate the cellular signaling.

\subsection{De Novo Detection Principles: Beyond the} Harmonic Oscillation-Based Mass Sensing

As demonstrated in Section 2.1.2, the fundamental physics for resonator-based sensing is the direct transduction of molecular chemisorption to a resonator surface into the resonant frequency shift. The detection principle described in Eq. (2) starts to break down when a resonator is scaled down to nanoscale. This is attributed to unanticipated effects such as surface effects. Specifically, when a device's dimension is significantly reduced, a surface-to-volume ratio is increased so as to increase the surface energy that is energetic cost to create a new surface due to scaling down. Because the surface stress is defined as a derivative of surface energy with respect to strain, the vibration behavior of nanoscale resonators is dominantly governed by surface effects $^{[28]}$. As a consequence, the resonance behavior of nanoscale resonator in response to molecular adsorption would be significantly affected by surface effects. Furthermore, the detection principle described in Eq. (2) holds for a case of harmonic oscillation. That is, if a nanoscale resonator vibrates in nonlinear flexural motion, a novel detection principle has to be enacted by including the effect of nonlinear oscillations. This section is restricted to descriptions on the fundamental physics for detection principles such as nonlinear oscillations and/or surface effects, albeit there are other possible effects such as coupling between mechanical motion and other physical quantities(e.g. electric field, optical property, etc.) may also play a critical role on the vibration behavior of nanoscale resonators.

\subsubsection{Nonlinear Oscillation-Based Detection}

When a resonator experiences a large amplitude- 
motion, in general, the resonance behavior is not well depicted by harmonic oscillation model due to geometric nonlinear effect ${ }^{[4-46]}$. For instance, a doubly-clamped beam exhibits the nonlinear flexural motion due to boundary conditions when a large amount of flexural motion is induced. The equation of motion for a doublyclamped resonator with atomic adsorption can be described as ${ }^{[47]}$

$$
\begin{aligned}
& E I \frac{\partial^{4} w(x, t)}{\partial x^{4}}-\left[T+\frac{E A}{2 L} \int_{0}^{L}\left\{\frac{\partial w(x, t)}{\partial x}\right\}^{2} d x\right] \frac{\partial^{2} w(x, t)}{\partial x^{2}} \\
& +\{\mu+\Delta \mu(x)\} \frac{\partial^{2} w(x, t)}{\partial t^{2}}=f(x, t)
\end{aligned}
$$

where $w(x, t)$ is a flexural deformation of a resonator, $x$ is a coordinate along the longitudinal direction of a resontoar, $t$ is a time, $E, I, A$, and $L$ represent the elastic modulus, cross-sectional moment of inertia, cross-sectional area, and length of a resonator, respectively, $T$ is a mechanical tension applied to the both ends of a resonator, $\mu$ is a mass per unit length for a resonator, i.e. $\mu=\rho A$ with $\rho$ being a mass density of a resonator, $\Delta \mu(x)$ is a mass per unit length for an adsorbed atomic layer, and $f(x, t)$ is an actuating force. In case of a uniform atomic adsorption over the entire length of a resonator, we have a relation of $\Delta \mu(x)=(\Delta m / L) \cdot H(x)$, where $\Delta m$ is the total mass of adsorbed atomic layer, and $H(x)$ is a Heaviside unit step function. When atomic adsorption occurs locally at $x=a$ (where $0<a<L$ ), we have $\Delta \mu(x)=\Delta m \cdot \delta(x-a)$, where $\delta(x)$ a Dirac delta function. Here, it should be noted that a term dictated by $(E A / 2 L) \int_{0}^{L}\{\partial w(x, t) / \partial x\}^{2} d x$ indicates the geometric nonlinear effect due to double-clamping boundary condition. When an actuating force is very small, the nonlinear effect can be easily neglected so that vibrational motion can be regarded as harmonic oscillation. However, in general, when actuating force is not negligible, nonlinear effect appears in vibrational motion. It should be noted that geometric nonlinearity-induced nonlinear oscillation induces the stiffening effect, that is, geometric nonlinearity produces the mechanical tension that increases the resonant frequency(see Fig. 6).

In order to solve the equation of motion depicted by Eq. (3), we have employed the Ritz method ${ }^{[48]}$ (or equivalently, galkerin's method $\left.{ }^{[49,50]}\right)$. Specifically, we assume that the deflection $w(x, t)$ is represented in the form of $w(x, t)=u(x) \cdot z(t)$, where $u(x)$ is an admissible deflection

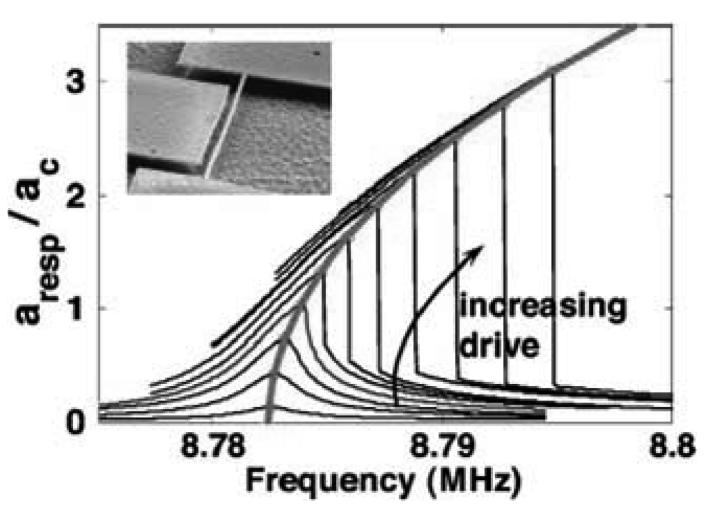

Fig. 6. Resonance response of nanowire resonator. As an actuation force increases, the resonance response becomes to exhibit the nonlinear response attributed to the geometric nonlinear effect due to doubly-clamped boundary condition. Reprinted with permission from ${ }^{[4]}$. Copyright 2006 American Institute of Physics.

eigenmode, and $z(t)$ is a vibration amplitude. We assume that $u(x)$ is given as $u(x)=(2 / 3)^{1 / 2}[1-\cos (2 \pi x /$ $L)$ ], which is the deflection eigenmode for a doublyclamped beam with neglecting the geometric nonlinear effect and mass adsorption. Further, it should be also noted that $u(x)$ satisfies the essential boundary conditions $u(0)=u(L)=u^{\prime}(0)=u^{\prime}(L)=0$, where a prime indicates the differentiation with respect to $x$. As a consequence, by substituting $w(x, t)=u(x) \cdot z(t)$ into Eq. (3) and subsequent integration with respect to $x$ by using integration by part, the equation of motion is transformed into Duffing equation ${ }^{[1,52]}$ given by

$$
\{\lambda+\eta T\} z(t)+\kappa[z(t)]^{3}+v \frac{d^{2} z(t)}{d t^{2}}=p_{0} \cos (\Omega t)
$$

where parameters for Duffing equation are well described in Ref. ${ }^{[47]}$. Here, $p_{0}$ and $\Omega$ indicate the actuating force and driving frequency(that is close to the resonant frequency), respectively. Fig. 6 shows the resonance behavior of doubly-clamped carbon nanotube(CNT) resonator. As shown in Fig. 6, as an actuating force increases, one can easily observe the nonlinear oscillation behavior of CNT resonator such that geometric nonlinear effect stiffens the CNT resonator so as to increase the resonant frequency of CNT resonator. Fig. 7 depicts the resonant frequency shift due to mass adsorption with respect to the total mass of adsorbed atoms as well as actuating force. It is interestingly and remarka- 
(a)

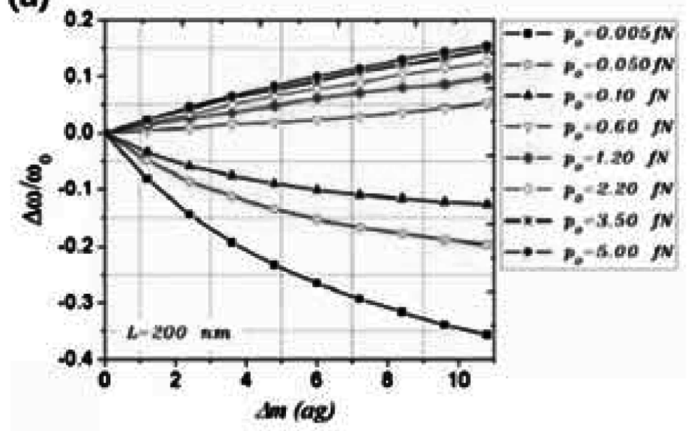

(b)

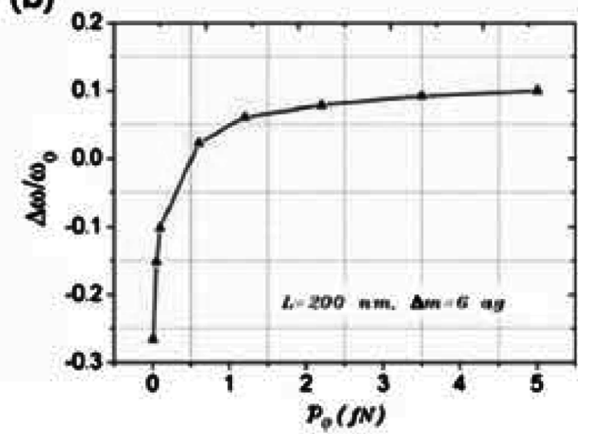

Fig. 7. Nonlinear oscillation-based nanomechanical mass sensing: (a) Resonant frequency shifts due to mass adsorption with respect to total added mass and actuation force. As an actuation force increases so as to induce the nonlinear oscillation, the atomic adsorption increases the resonant frequency, which is contrast to the detection principle based on harmonic oscillation-based mass sensing. (b) Resonant frequency shift due to mass adsorption with added mass of $\sim 6$ ag as a function of actuation force. When an actuation force is larger than $\sim 1 \mathrm{fN}$, the nonlinear oscillation-driven increase in the resonant frequency due to mass adsorption can be observed. Reprinted with permission from ${ }^{[47]}$. Copyright 2009 American Institute of Physics.

bly found that, in case of nonlinear oscillation regime, mass adsorption increases the resonant frequency of CNT resonator, which is quite different from harmonic oscillation-based mass sensing. In order to gain a deeper insight into the role of nonlinearity on the mass sensitivity, we have considered the resonant frequency shift for CNT resonator due to mass adsorption with the amount of $6 \mathrm{ag}$ as a function of actuating force(see Fig. 7). It is shown that, when actuating force is close to $\sim 1 \mathrm{fN}$, one can observe the unique mass-sensing behavior due to nonlinear oscillation. This indicates that nonlinear oscillation-based unique mass-sensing characteristics can be easily observed in doubly-clamped resonator even when an actuating force with the amount of $\sim 1 \mathrm{fN}$ is applied to a resonator. We believe that nonlinear oscillationbased mass-sensing may be a viable, important route to improve the detection sensitivity for resonator-based detections.

\subsubsection{Surface Stress Effect on Resonator-Based} Sensing

As stated above, as a resonator is scaled down to nanoscale, the resonator can be characterized by a large surface-to-volume ratio, which implies that surface energy plays a dominant role on the deformation mechanism for such a resonator. In other words, the surface effect such as surface stress plays a critical role on the dynamic behavior of nanoresonators. Moreover, molecular adsorp- tion onto a resonator's surface induces the change of surface state, which may generate the surface stress change that may shift the resonant frequency of a resonator.

In an earlier work by Thundat and coworkers ${ }^{[53]}$, it is provided that atomic adsorption induces the surface stress change that critically shifts the resonant frequency of a microcantilever. In the similar spirit, recent studies ${ }^{[54-56]}$ have reported that the surface stress change due to biomolecular adsorption significantly induces the resonant frequency shift for a microcantilever. In order to understand the role of surface stress on the resonant frequency-behavior of microcantilever, the continuum mechanics model has been suggested ${ }^{[54-56]}$.

$$
E I \frac{\partial^{4} w(x, t)}{\partial x^{4}}+\frac{\partial}{\partial x}\left[\tau x \frac{\partial w(x, t)}{\partial x}\right]+\rho A \frac{\partial^{2} w(x, t)}{\partial t^{2}}=0
$$

where $w(x, t)$ is a bending deflection of a microcantilever, $E, I, \rho$, and $A$ indicate the elastic modulus, crosssectional moment of inertia, mass density, and crosssectional area of a microcantilever, respectively, and $\tau$ is a constant surface stress(that is due to atomic adsorption). Here, we assume that resonance behavior of a microcantilever obeys a harmonic oscillation, because cantilevered boundary condition does not induce the geometric nonlinear effect. With assumption of $w(x, t)=u(x) \cdot \exp [i \omega t]$, where $\omega$ and $u(x)$ represent the resonant frequency and its corresponding deflection eigenmode, the equation of motion becomes the eigen- 
value problem as follows:

$$
E I \frac{d^{4} u}{d x^{4}}+\frac{d}{d x}\left(\tau x \frac{d u}{d x}\right)=\rho A w^{2} u
$$

The analytical solution to the eigenvalue problem depicted as Eq. (6) provides the relationship between constant surface stress and surface stress-induced resonant frequency shift such as ${ }^{[55]}$

$$
\frac{\Delta \omega}{\omega_{0}}=\frac{\tau L^{3}}{\pi^{2} E I}
$$

where $\omega_{0}$ and $\Delta \omega$ indicate the resonant frequency of a bare cantilever(i.e. without surface stress) and the resonant frequency shift due to constant surface stress, respectively.

Even though a theoretical model depicted by Eq. (5) has been extensively employed to characterize the resonant frequency shift due to surface stress change driven by atomic adsorption, the model is essential incorrect in that it violates the Newton's third law. Specifically, when a constant surface stress is applied on the surface of an elastic body, such a constant surface stress induces the residual stress for a cross-sectional area in order to satisfy the force equilibrium condition. Gurtin and coworkers ${ }^{[57]}$ showed that the residual stress due to constant surface stress cancels out the effect of constant surface stress in the bending deformation of a beam. In other words, constant surface stress does not affect the resonance behavior of a mechanical beam. Recently, Sader and a coworker ${ }^{[58,59]}$ have pointed out the incorrect assumptions in one-dimensional beam model and suggested a three-dimensional plate model in order to understand the role of a constant surface stress on the resonance behavior of a microcantilever. Sader's model provides the relationship between a constant surface stress and the resonant frequency shift in the form of ${ }^{[88]}$

$$
\frac{\Delta \omega}{\omega_{0}}=\phi(v)\left[\frac{(1-v) \tau}{E h}\right]\left(\frac{b}{L}\right)\left(\frac{b}{h}\right)^{2}
$$

Here, $\phi(v)$ is a function with respect to Poisson's ratio $v$, and a cantilever has a dimension of $L \times b \times h$ (length $\times$ width $\times$ thickness). It can be easily shown that, in case of a one-dimensional beam(i.e. $b \rightarrow 0$ ), the resonant frequency shift due to constant surface stress converges to zero. This indicates that Sader's model does not violate the Gurtin's argument, i.e. Sader's model satisfies the equilibrium condition. Sader and a coworker have revisited the experimental works ${ }^{[7,54,55]}$ on the atomic adsorption onto a microcantilever in order to understand the resonant frequency shift due to surface stress originating from atomic adsorption. They have argued that, based on their model given by Eq. (8), a constant surface stress is insufficient to describe the resonant frequency shift due to atomic adsorption. One of possible factors for atomic adsorption-induced frequency shift is the adsorption-induced change of surface elastic stiffness that can also affect the resonant frequency of a resonator ${ }^{[26]}$. This has not been extensively considered for gaining insight into resonant frequency shift due to atomic adsorption except a recent study by Lu et al. ${ }^{[8]}$. Moreover, a continuum mechanics-based model may be insufficient to provide a fundamental insight into the role of atomic adsorption on the resonance behavior of a resonator. Recently, a multiscale modeling concept ${ }^{[60-63]}$ has been introduced in such a way that a resonator is modeled as a continuous body while an adsorbed molecule is modeled as atomic model. This multiscale modeling concept is now received much attention for its ability to describe the resonance behavior of a device in response to atomic adsorption in viewpoint of atomic scale, albeit such a multiscale modeling concept is still lack for the case of biomolecular adsorption onto a resonator.

\section{Molecular Detection using Nanowire Field Effect Transistor}

The ability to sense minute concentrations of biomolecules is critical for the biotechnological researches and developments including proteomics and genomics ${ }^{[64]}$. Since Cui et al, reported in 2001 that nanowires(NWs) can be utilized as building blocks of nanosensor for detection of biological and chemical species, the nanowire field effect transistor(NW-FET) becomes one of the most promising platform for ultra-high sensitive, real-time and label-free detection of the target biomolecule $^{[65]}$. The unlabeled sensing ability of the NW-FET platform eliminates issues of attaching signal-generating labels to target molecules and complex optical systems, thus allowing for simple, rapid, and portable detection $^{[66-68]}$. In addition, one-dimensional morphology of the NWs overcomes sensitivity limitations of microplanar FETs since binding of charged target molecules to the modified surface of the NW leads to accumulation 


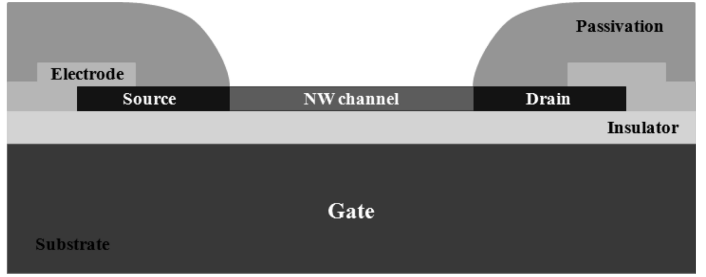

Fig. 8. Structure of an NW-FET biosensor(cross sectional view).

or depletion of charge carriers through the entire cross section, rather than only in the surface region of the microplanar FETs ${ }^{[67,69]}$.

\subsection{Structure of NW FETs}

Compared to microplanar FETs ${ }^{[70]}$, most of NW-FET has utilized three common electrodes for detection of biological species. Fig. 8 shows the typical structure of an NW-FET sensor. Source and drain are connected through semiconductor NWs. In general, the gate is separated from the semiconductor NW by insulators. Incase of using silicon-on-insulator(SOI) wafer that is the most widely used substrate, the handle $\mathrm{Si}$ substrate is commonly used as the back gate.

\subsection{Detection Mechanism of NW FET Biosensors}

In order to sense the specific target molecule, biorecognition element(receptor) should be anchored to the NW surface. Depending on the type of bio-recognition element, there are immunologically modified FETs, DNA-modified FETs, cell-based FETs and enzymemodified FETs ${ }^{[66]}$. Binding of the target molecule to the modified NW surface modulates the conductance of the NW channel. Like semiconductor, NWs can be divided into two groups depending on the majority carriers in the NW. If majority carriers are holes the NW semiconductor is p-type, and if electrons are the main carriers it is an n-type semiconductor NW. The carrier concentration in the NW is proportional to the conductance of the channel and inversely proportional to resistance of the channel, which affects the source-drain current. For the detection of target biomolecules the source-drain current was measure at a fixed applied voltage between source-drain electrodes. Also, the applied gate voltage is constant over time. Fig. 9 shows response of the NWFET to a target molecule. If the target biomolecule is positively charged and bound to the modified NW sur-

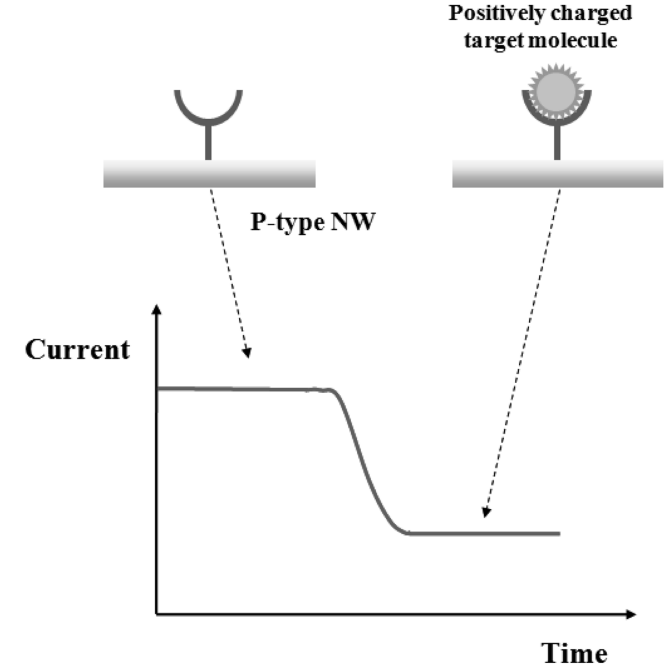

(a)

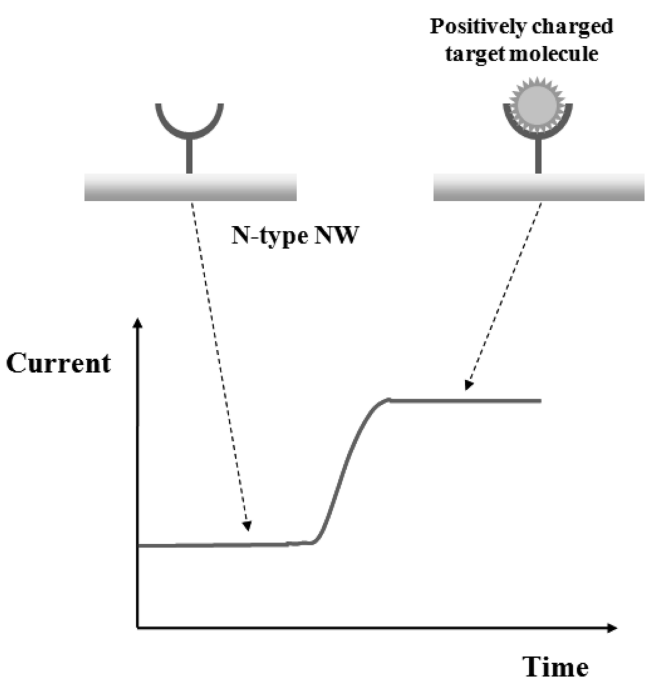

(b)

Fig. 9. Sensing mechanism of semiconductor NW FET: (a). Conductance change of p-type NW FET due to binding of positively charged target molecule. (b) Conductance change of n-type NW FET due to binding of positively charged target molecule.

face of a p-type semiconductor, the source-drain current decreases. It is equivalent to applying a positive voltage to the gate electrode of the p-channel MOSFET(metaloxide-semiconductor FET), resulting in a decrease of 
the channel conductance due to depletion of the majority carriers(see Fig. 9(a)). If electrons are majority carriers and the bound target biomolecule is positively charged, the source-drain current will increase. This is caused by accumulation of majority carriers(electrons) in NWs(see Fig. 9(a)). Complementary detection of the target molecule using both p-type and n-type NW-FET can reduce false positive signals generated from electrical noise and/or non-specific bindings. Thus, real signals have to show a reversible conductance increase and decrease using both p-type and n-type NW-FET ${ }^{[69]}$.

\subsection{Materials of NW FET Including SiNW}

Materials of the NWs are $\mathrm{Si}, \mathrm{Ge}, \mathrm{In}_{2} \mathrm{O}_{3}, \mathrm{SnO}_{2}, \mathrm{ZnO}$ etc. In particular, SiNWs have gained wide attentions since they can be prepared by both "botton-up" and “top-down" paradigms ${ }^{[65,67,69,71]}$. NWs produced by "bottom-up" approaches can be prepared by chemical vapor deposition(CVD), vapor-liquid-solid(VLS) synthesis and laser ablation VLS ${ }^{[6,72,73]}$. The SiNWs synthesized by CVD using gold nanoclusters $(\sim 20 \mathrm{~nm})$ as catalysts were dispersed on the silicon substrate with thin insulator such as a silicon dioxide layer. Then, source and drain were formed by metal( $\mathrm{Ti} / \mathrm{Au})$ contact. Finally, a passivation layer was deposited ${ }^{[72,73]}$. The "bottom-up" approach can produce high-quality NWs; however, this approach involves "pick and place" techniques which reflect poor device uniformity ${ }^{[67]}$. On the other hand, the "top-down" approach reduced their bulk dimensions to fabricate nanodevices. NW-FET devices were fabricated from SOI wafers. NW patterns from the Si device layer were usually generated by e-beam lithography. The patterned active Si device layer with NW, source and drain was then etched away. The resistivity of the NW can be modulated by control of implantation dose. Protection layer was then deposited except the NW surface ${ }^{[11,74-77]}$. Fig. 10 shows a fully fabricated SiNW FET using the top-down approach. The NW area was exposed to chemical solutions for modification of the surface and detection of the target molecules. This technique can be CMOS compatible and provide precise control of positioning and integration of circuits and sensor arrays, producing high yield and large scale fabrication ${ }^{[67,70,71]}$.

\subsection{Surface modification of SiNW FET}

Topics of surface modification will be limited to

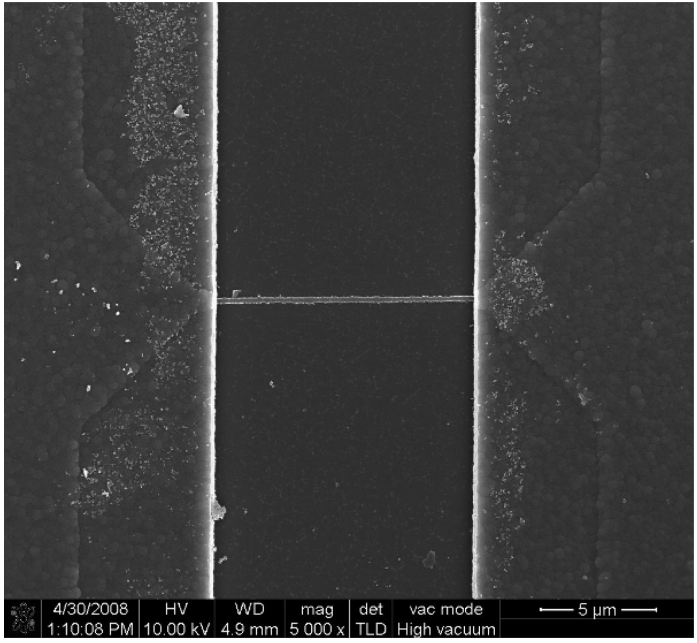

Fig. 10. Fully fabricated SiNW FET using a top-down approach.

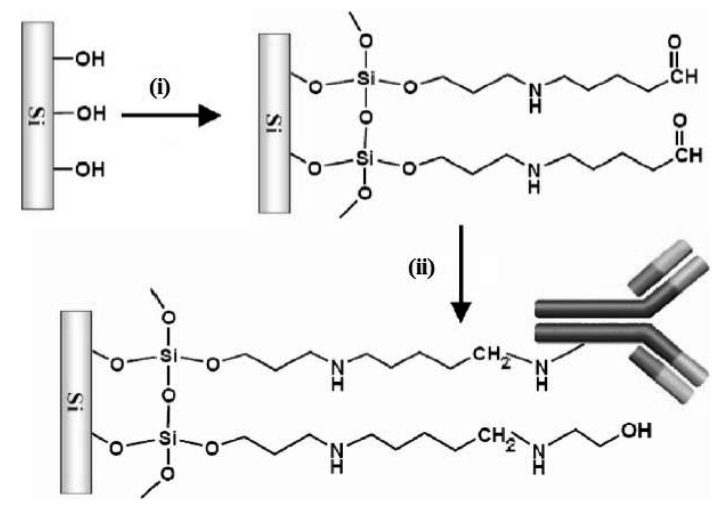

Fig. 11. Chemical process to anchor biorecognition elements to the NW surface: Sequence reaction of $1 \%$ APTES in ethanol and $25 \mathrm{wt} \%$ glutaraldehyde with $\mathrm{NaBH}_{3} \mathrm{CN}$ (i) provided aldehyde-functionalized surface. Antibody with $\mathrm{NaBH}_{3} \mathrm{CN}$ to immobilize a biorecognition elements and ethanolamine with $\mathrm{NaBH}_{3} \mathrm{CN}$ to block non-specific binding were injected. Reprinted with permission from ${ }^{[71]}$. Copyright 2007 American Institute of Physics.

immunodetections. To functionalize the device for the detection of the target molecule, the NW surface has to be modified with biorecognition elements so as to acquire selectivity. In general, a linker with two functional termini is used to immobilize recognition element on the NW surface. The most common linkers to functionalize a native oxide coated SiNWs are alkoxysilane 
derivatives such as 3-(trimethoxysilyl)propylaldehyde (APTMS $)^{[66,72,73]}$. Immobilization procedure involving the use of APTMS is as follows. Firstly, an oxygen plasma treatment was performed to form $\mathrm{OH}$-modified surface. Reaction of the OH-modified surface with the APTMS provides an aldehyde-silane-modified SiNW surface. Then, a solution containing a bio-receptor such as an antibody was drawn to the modified SiNW surface. After the covalent immobilization of the bio-receptor, unreacted sites of the linker was usually blocked by the chemicals such as ethanolamine, 2-mercaptoethanol, Tris buffer and so forth ${ }^{[66,71-73]}$. Another popular linker is 3aminopropyltriethoxysilane(APTES) ${ }^{[64,71]}$. After sequence reactions of the $\mathrm{SiNW}$ with $\mathrm{O}_{2}$ plasma,exposure of APTES in ethanol to the OH-modified surface enables the NW surface to form $-\mathrm{NH}_{2}$ group, which could be activated toward biorecognition layer by a proper reagent. Kim et al.utilized glutaraldehyde to convert the surface with $-\mathrm{NH}_{2}$ to the aldehyde-functionalized surface since the amine groups of L-lysine in antibody of prostate specific antigen(PSA) can easily react with the aldehyde(see Fig. 11) ${ }^{[71]}$. After attachment of the biorecognition element(monoclonal anti-PSA), they also used the ethanolamine to quench the unreacted aldehyde groups to prevent non-specific adsorption. During the reactions of the aldehyde-modified surface with the biorecognition element and/or ethanolamine, cyanoborohydride $\left(\mathrm{NaBH}_{3} \mathrm{CN}\right)$ was added to enhance the reactions.

\subsection{Delivery of Sample}

Detection of target molecules using NW FETs is carried out in wet state. Thus, the delivery system of the analyte solution is another important factor to be considered since binding of the target molecule to the modified surface is affected by the delivery methods and geometry of the fluidic device. Microfluidic channels ${ }^{[67,71]}$ and mixing cell $^{[74]}$ have been widely used for such a delivery. One of the advantages of using microfluidic systems is that the analysis can be carried out using extremely small sample volume on the order of a nanoliter. In microfluidics, the flow is laminar, and the main mixing mechanism is to be diffusion. The most popular material for such a microfluidics is polydimethylsiloxane(PDMS). It can be molded using SU-8 which is a negative photoresist suitable for high-aspect-ratio structure. Microchannels are widely used for the delivery of the target analyte(see Fig. 12(a)). However, the diffusion-based mixing may limit on

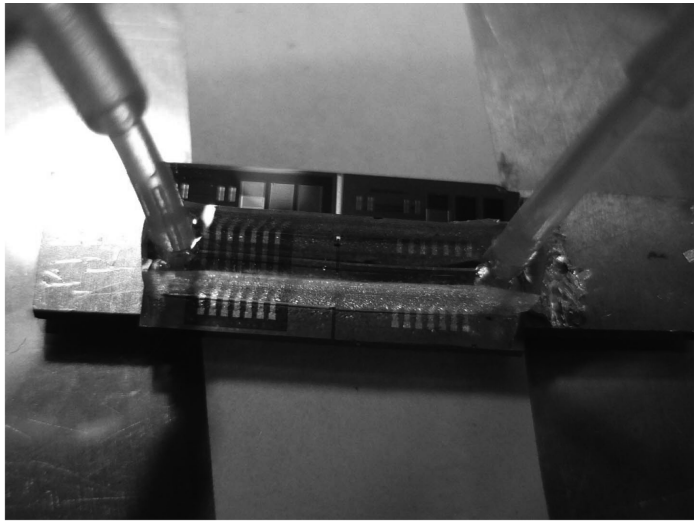

(a)

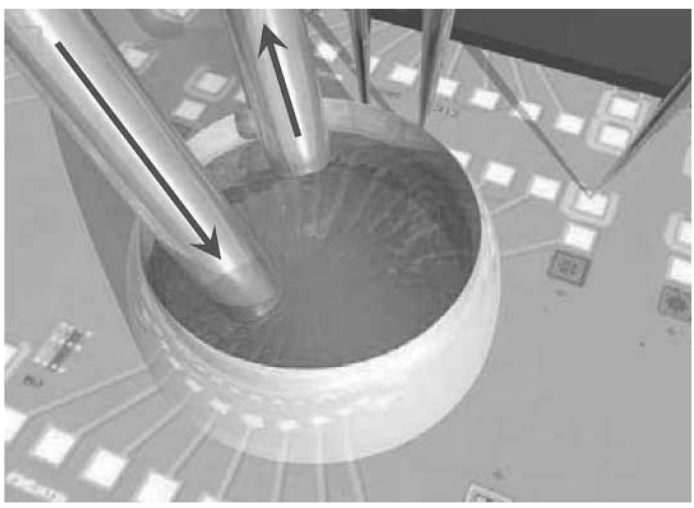

(b)

Fig. 12. Microfluidics for sample delivery: (a) PDMS microfludic channel was mounted on the NW FET device, and (b) a cell based on a fluid exchange induced mixing on the top of the NW. (b) Reprinted with permission from Macmillan Publisher Ltd: Nature ${ }^{[7]}$, copyright 2007.

response time of the sensor. Stern et al. designed a simple macro-scale chamber to overcome diffusion-limited systems. It can induce mixing during fluid exchange(see Fig. $12(b))^{[74]}$. In general, sealing of the microfluidic structure is performed using $\mathrm{O}_{2}$-plasma.

\subsection{Debye Length}

An important factor to be considered for the electrical detection of the target molecule is the Debye length $\left(\lambda_{\mathrm{D}}\right)$. The Debye length is defined as the maximum distance at which an external charge can affect the device performance. Since the size of the bound receptor proteins from the sensor surface is a few nanometers, $\lambda_{D}$ 


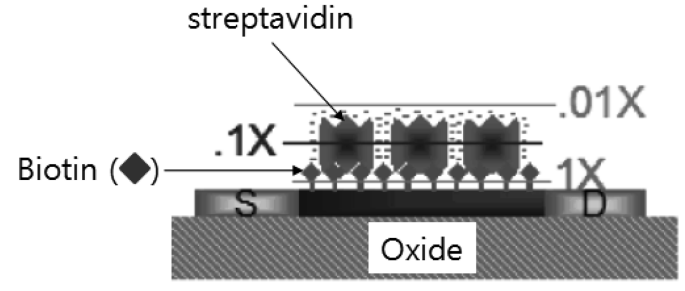

(a)

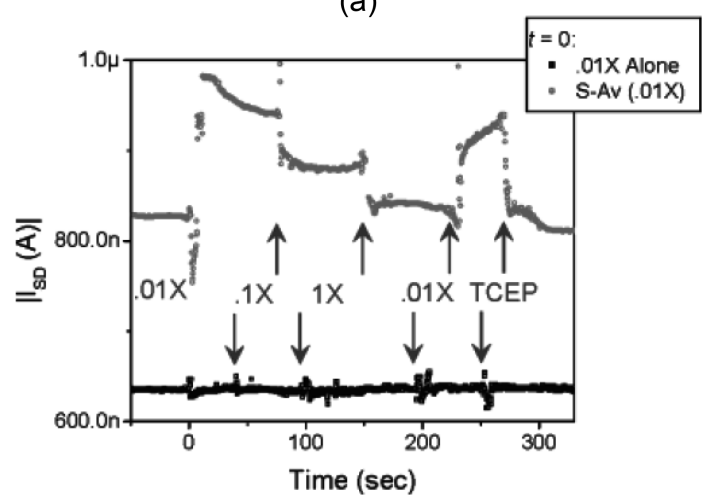

(b)

Fig. 13. Impact of Debye screening: (a) Schematic showing from the device surface: S and D stand for source and drain, respectively. The purple diamonds are biotin, and the red objects are steptavidin. The $1 \times$ line represents the screening length $\left(\lambda_{\mathrm{D}}\right)$ from $1 \times$ PBS relative to the protein, and the $0.1 \times$ and $0.01 \times$ lines represent that from 1:10 and 1:100 dilutions of this buffer, respectively. (b) Biotin-functionalized sensor response $\left(\left|I_{\mathrm{SD}}\right|\right.$ vs. time) to varying buffer ionic concentrations with (red) and without(black) streptavidin addition at time $=0$. The blue text gives the PBS buffer concentration, and the blue arrows represent the onset of solution exchange. Reprinted with permission from ${ }^{[64]}$. Copyright 2007 American Chemical Society.

should be carefully considered for optimal detection of the target molecule. The charge of the target molecules can be screened by dissolved solution counterions ${ }^{[64]}$. The Debye length in water can be approximated using the formula $\lambda_{\mathrm{D} \propto}(I)^{-1 / 2}$, where $I$ is the ionic strength of the buffer solution ${ }^{[66]}$. Stern et al. demonstrated that increasing buffer ionic strengths decreased $\lambda_{\mathrm{D}}$, which influence on the device sensitivity. For these studies, they used the well-studied biotin-streptavidin ligandreceptor system(see Fig. 13(a)). In figure f, an isoelectric point $(\mathrm{pI})$ of the streptavidin is $\sim 5.6$, and the $\mathrm{NW}$ device was p-type. Thus, binding of the streptavidin to biotin-functionalized NW surface resulted in an increased $\left|\mathrm{I}_{\mathrm{SD}}\right|$. An increase in the ionic strength of the buffer could screen the charge of the target molecule more efficiently(see Fig. 13(b)). If the ionic strengths of the buffer are $0.01,0.1$, and 1 ' PBS, then $\lambda_{\mathrm{D}}$ would be approximately $\sim 7.3, \sim 2.3$, and $0.7 \mathrm{~nm}$. Thus optimal ionic strength is required for the best detection of the target molecule by suppressing other electrolytes in the buffer and avoiding an effective screen of the charged target molecule ${ }^{[64]}$.

\subsection{Label-free and Real-time Electrical Detection} of Analytes

Lieber's group has detected various biomolecules such as proteins, nucleic acids, small molecules and even viruses utilizing as-grown $\mathrm{SiNWs}^{[65,69,72,73]}$. Cui et $a l$. functionalized p-type SiNWs with biotin. The conductance of the p-type SiNW increased with binding of streptavidin to biotin-modified surface since streptavi$\operatorname{din}(\mathrm{pI} » 5$ to 6$)$ is negatively charged at the $\mathrm{pH}$ of their measurement. They detected streptavidin down to concentration of $10 \mathrm{pM}^{[65]}$. Wang et al. demonstrated that APT binding to Abl-modified p-type SiNW can be distinguished above background at concentration as low as $100 \mathrm{pM}$. The conductance also increased with binding of negatively charged ATP to Abl ${ }^{[72]}$.

Stern et al.fabricated a SiNW FET-based biosensor by the top-down approach with e-beam lithography and anisotropic Si etching. They detected $100 \mathrm{fM}$ concentration of mouse immunoglobulin $\mathrm{G}(\mathrm{IgG})$ and immunoglobulin A (IgA) under conditions closer to physiological serum(see Fig. 14(a) and (b)). They also measured the device sensitivity with various solution $\mathrm{pH}$ since the effective charge of the target molecule can be affected by solution $\mathrm{pH}$. The effective charge of avidin can be decreased by increasing buffer solution from $\mathrm{pH} 7.4$ to $\mathrm{pH} 10.5$ since pI of avidin is »10.5(see Fig. 14(c)) [74]. Kim et al. has demonstrated detection of PSA of $1 \mathrm{fg} / \mathrm{ml}$ concentration using an n-type SiNW FET which was patterned by e-beam lithography(see Fig. 15(a)). They defined the sensitivity as $\left[G_{O}-G_{P S A}\right] / G_{O}$, where $G_{O}$ is the conductance after the injection of the buffer solutions and $\mathrm{G}_{\mathrm{PSA}}$ the conductance after the injection of the PSA. They investigated the effect of the SiNW size and doping concentrations on the sensitivity. The lower doping concentration of SiNW increased the sensitivity(see Fig. 


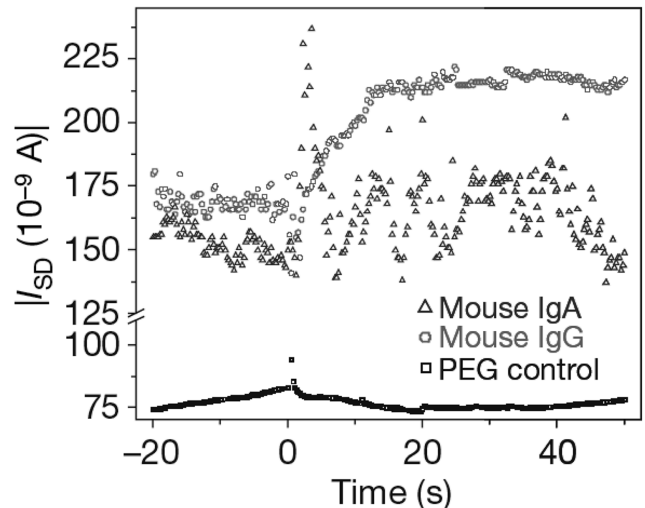

(a)

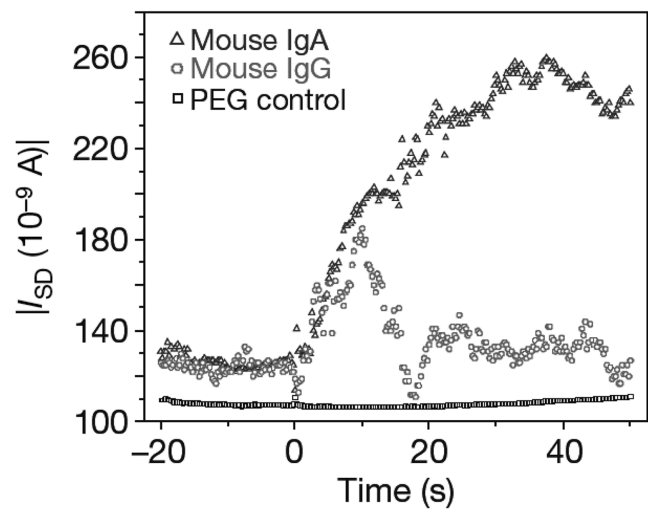

(b)

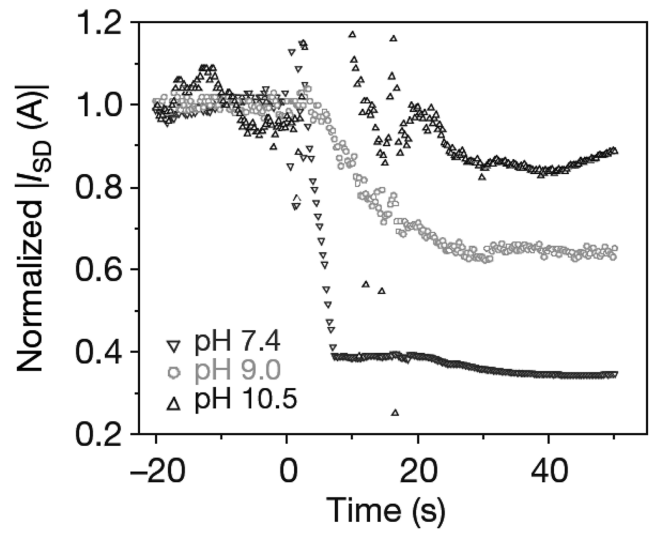

(c)

Fig. 14. Sensor responses to $100 \mathrm{fM}$ mouse-IgG(red) or 100 $\mathrm{fM}$ mounse-IgA(blue) for (a) goat anti-mouse IgGfunctionalized sensor and (b) goat anti-mouse IgAfunctionalized sensor. (c) Sensor response to $1 \mathrm{nM}$ avidin in buffer with different $\mathrm{pH}$ values to biotinylated sensor. Reprinted with permission from Macmillan Publisher Ltd: Nature ${ }^{[74]}$, copyright 2007.

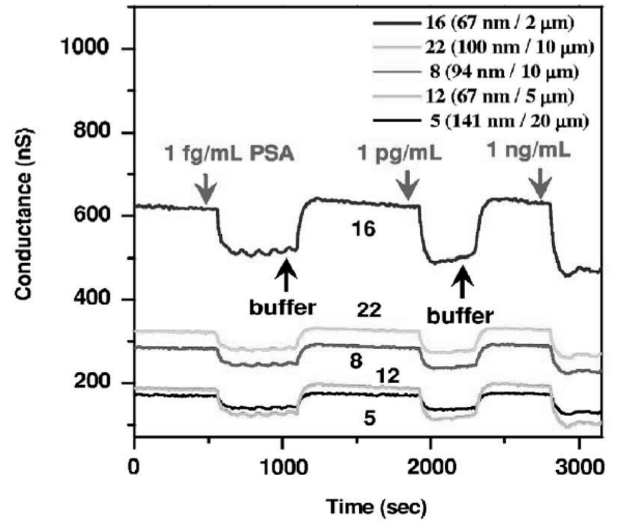

(a)

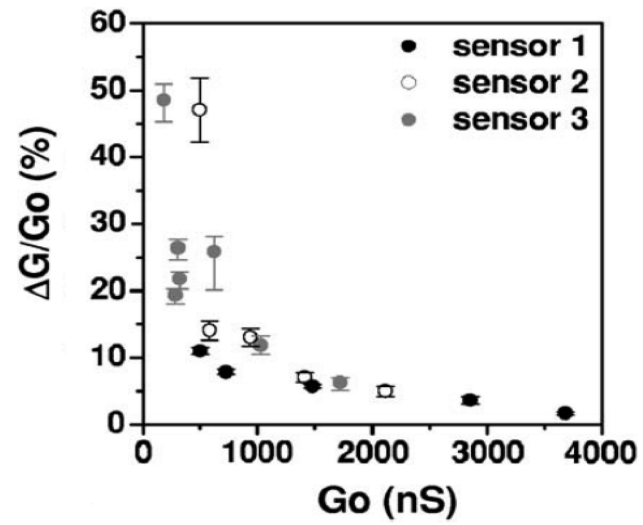

(b)

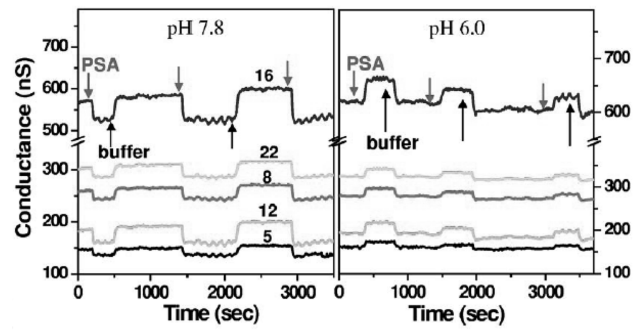

(c)

Fig. 15. Detection of PSA: (a) Conductance vs. time with five different n-type SiNW FETs simultaneously after reversible injection of buffer and $1 \mathrm{fg} / \mathrm{ml}$, $1 \mathrm{pg} / \mathrm{ml}$, and $1 \mathrm{ng} / \mathrm{ml}$ of PSA concentrations. The numbers in the box represent channel length and width. (b) Sensitivity vs. channel conductance. The doping concentration of sensor 1 is $1 \times$ $10^{19} \mathrm{~cm}^{-3}$, and that of sensors 2 and 3 is $3 \times 10^{18} \mathrm{~cm}^{-3}$. (c) Conductance vs. time with five different devices after reversible injection of difference buffers with $\mathrm{pH} 7.8$ and $\mathrm{pH}$ 6.0. Reprinted with permission from ${ }^{[71]}$. Copyright 2007 American Institute of Physics. 
15(b)). They also measured the response of NW-FET to the target molecule with different buffer solution $\mathrm{pH}$. The conductance increased at $\mathrm{pH}$ above $\mathrm{pI}$ and decreased at $\mathrm{pH}$ below $\mathrm{pI}$ of PSA(pI » 6.9) as expected (see Fig. 15(c)). They also concluded the device sensitivity can be improved by reducing dimensions and doping concentration of the NWs. Their result is the highest sensitivity reported to date ${ }^{[71]}$.

\section{Conclusion}

In this review, we have provided the basic concepts and current state-of-art in the de novo label-free biosensing based on nanoresonators and nanowires. Our review suggests a great potential of nanoresonators and nanowires for label-free detection with high sensitivity. In particular, the sensor based on nanoresonators and nanowires allows the early diagnostics of diseases with high portability and efficiency. Furthermore, as scaled down to nanometer resolutions, the sensor experience the unique, unanticipated physical phenomena, which implies that nanosensor can be a toolkit for studying the nanoscale physics. For instance, as discussed in our review, the dynamic behavior of nanoresonators is quite unique due to surface effects and/or nonlinear effects. Furthermore, this unique, nanoscale physical phenomena observed in nanosensors allows us to develop the novel sensing paradigm for future diagnostics, drugscreening, and environmental monitoring.

\section{Acknowledgement}

This research was supported by Basic Science Research Program through the National Research Foundation of Korea(NRF) funded by the Ministry of Education, Science and Technology under Grant No. NRF2010-0015527, NRF-2009-0071246, and NRF-2008313-D00031.

\section{References}

[1] M. A. Cooper Edit., Label-free biosensors, Cambridge, New York, 2009.

[2] L. G. Carrascosa, M. Moreno, M. Álvarez, and L. M. Lechuga, "Nanomechanical biosensors: a new sensing tool," Trac-Trends Anal. Chem., vol. 25, pp. 196-206, 2005.
[3] S. Choi, Y. Yang, and J. Chae, "Surface plasmon resonance protein sensor using vroman effect," Biosens. Bioelectron., vol. 24, pp. 893-899, 2008.

[4] Y. T. Yang, C. Callegari, X. L. Feng, K. L. Ekinci, and M. L. Roukes, "Zeptogram-scale nanomechanical mass sensing," Nano Lett., vol. 6, pp. 583-586, 2006.

[5] M. Li, H. X. Tang, and M. L. Roukes, "Ultra-sensitive NEMS-based cantilevers for sensing, scanned probe and very high-frequency applications," Nat. Nanotechnol., vol. 2, pp. 114-120, 2007.

[6] K. Jensen, K. Kim, and A. Zettl, "An atomic-resolution nanomechanical mass sensor," Nat. Nanotechnol., vol. 3, pp. 533-537, 2008.

[7] J. Lagowski, H. C. Gatos, and J. E. S. Sproles, "Surface stress and the normal mode of vibration of thin crystals : GaAs," Appl. Phys. Lett., vol. 26, pp. 493495, 1975.

[8] P. Lu, H. P. Lee, C. Lu, and S. J. O'Shea, "Surface stress effects on the resonance properties of cantilever sensors," Phys. Rev. B., vol. 72, pp. 085405 , 2005.

[9] K. Eom, J. Yang, J. Park, G. Yoon, Y. Sohn, S. Park, D. Yoon, S. Na, and T. Kwon, "Experimental and Computational Characterization of Biological Liquid Crystals: A Review of Single-Molecule Bioassays," Int. J. Mol. Sci., vol. 10, pp. 4009-4032, 2009.

[10] D. J. Muller and Y. F. Dufrene, "Atomic force microscopy as a multifunctional molecular toolbox in nanobiotechnology," Nat. Nanotechnol., vol. 3, pp. 261-269, 2008.

[11] K. M. Goeders, J. S. Colton, and L. A. Bottomley, "Microcantilevers: Sensing Chemical Interactions via Mechanical Motion," Chem. Rev., vol. 108, pp. 522-542, 2008.

[12] G. G. Stoney, "The tension of metallic films deposited by electrolysis," Proc. R. Soc. Lond. A, vol. 82, pp. 172-175, 1909.

[13] R. Berger, E. Delamarche, H. P. Lang, C. Gerber, J. K. Gimzewski, E. Meyer, and H. J. Guntherodt, "Surface stress in the self-assembly of alkanethiols on gold," Science, vol. 276, pp. 2021-2024, 1997.

[14] T. Kwon, J. Park, J. Yang, D. S. Yoon, S. Na, C.W. Kim, J. S. Suh, Y. M. Huh, S. Haam, and K. Eom, "Nanomechanical in situ monitoring of proteolysis of peptide by cathepsin B," PLoS ONE, vol. 4, pp. e6248, 2009.

[15] K. Castelino, B. Kannan, and A. Majumdar, "Characterization of grafting density and binding efficiency of DNA and proteins on gold surfaces," 
Langmuir, vol. 21, pp. 1956-1961, 2005.

[16] T. Y. Kwon, K. Eom, J. H. Park, D. S. Yoon, T. S. Kim, and H. L. Lee, "In situ real-time monitoring of biomolecular interactions based on resonating microcantilevers immersed in a viscous fluid," Appl. Phys. Lett., vol. 90, pp. 223903, 2007.

[17] P. S. Waggoner and H. G. Craighead, "Micro- and nanomechanical sensors for environmental, chemical, and biological detection," Lab Chip, vol. 7, pp. 1238-1255, 2007.

[18] G. H. Wu, R. H. Datar, K. M. Hansen, T. Thundat, R. J. Cote, and A. Majumdar, "Bioassay of prostatespecific antigen(PSA) using microcantilevers," Nat. Biotechnol., vol. 19, pp. 856-860, 2001.

[19] J. Zhang, H. P. Lang, F. Huber, A. Bietsch, W. Grange, U. Certa, R. McKendry, H. J. Guntherodt, M. Hegner, and C. Gerber, "Rapid and label-free nanomechanical detection of biomarker transcripts in human RNA," Nat. Nanotechnol., vol. 1, pp. 214220, 2006.

[20] J. Fritz, M. K. Baller, H. P. Lang, H. Rothuizen, P. Vettiger, E. Meyer, H. J. Guntherodt, C. Gerber, and J. K. Gimzewski, "Translating biomolecular recognition into nanomechanics," Science, vol. 288, pp. 316-318, 2000.

[21] R. McKendry, J. Y. Zhang, Y. Arntz, T. Strunz, M. Hegner, H. P. Lang, M. K. Baller, U. Certa, E. Meyer, H. J. Guntherodt, and C. Gerber, "Multiple label-free biodetection and quantitative DNA-binding assays on a nanomechanical cantilever array," Proc. Natl. Acad. Sci. USA., vol. 99, pp. 9783-9788, 2002.

[22] G. H. Wu, H. F. Ji, K. Hansen, T. Thundat, R. Datar, R. Cote, M. F. Hagan, A. K. Chakraborty, and A. Majumdar, "Origin of nanomechanical cantilever motion generated from biomolecular interactions," Proc. Natl. Acad. Sci. USA., vol. 98, pp. 1560-1564, 2001.

[23] J. W. Ndieyira, M. Watari, A. D. Barrera, D. Zhou, M. Vogtli, M. Batchelor, M. A. Cooper, T. Strunz, M. A. Horton, C. Abell, T. Rayment, G. Aeppli, and R. A. McKendry, "Nanomechanical detection of antibiotic-mucopeptide binding in a model for superbug drug resistance," Nat. Nanotechnol., vol. 3, pp. 691-696, 2008.

[24] T. Braun, V. Barwich, M. K. Ghatkesar, A. H. Bredekamp, C. Gerber, M. Hegner, and H. P. Lang, "Micromechanical mass sensors for biomolecular detection in a physiological environment," Phys. Rev. E., vol. 72, pp. 031907, 2005.

[25] D. W. Chun, K. S. Hwang, K. Eom, J. H. Lee, B.
H. Cha, W. Y. Lee, D. S. Yoon, and T. S. Kim, "Detection of the Au thin-layer in the Hz per picogram regime based on the microcantilevers," Sens. Actuat. A, vol. 135, pp. 857-862, 2007.

[26] W. Haiss, "Surface stress of clean and adsorbatecovered solids," Rep. Prog. Phys., vol. 64, pp. 591, 2001.

[27] H. Ibach, "The role of surface stress in reconstruction, epitaxial growth, and stabilization of mesoscopic structures," Surf. Sci. Rep., vol. 29, pp. 193263, 1997.

[28] L. B. Freund and S. Suresh, Thin Film Materials, Cambridge University Press, Cambridge, 2003.

[29] B. Ilic, Y. Yang, K. Aubin, R. Reichenbach, S. Krylov, and H. G. Craighead, "Enumeration of DNA molecules bound to a nanomechanical oscillator," Nano Lett., vol. 5, pp. 925-929, 2005.

[30] T. P. Burg, M. Godin, S. M. Knudsen, W. Shen, G. Carlson, J. S. Foster, K. Babcock, and S. R. Manalis, "Weighing of biomolecules, single cells and single nanoparticles in fluid," Nature, vol. 446, pp. 1066-1069, 2007.

[31] A. K. Bryan, A. Goranov, A. Amon, and S. R. Manalis, "Measurement of mass, density, and volume during the cell cycle of yeast," Proc. Natl. Acad. Sci. USA., vol. 107, pp. 999-1004, 2010.

[32] K. Y. Gfeller, N. Nugaeva, and M. Hegner, "Micromechanical oscillators as rapid biosensor for the detection of active growth of escherichia coli," Biosens. Bioelectron., vol. 21, pp. 528-533, 2005.

[33] N. Nugaeva, K. Y. Gfeller, N. Backmann, H. P. Lang, M. Duggelin, and M. Hegner, "Micromechanical cantilever array sensors for selective fungal immobilization and fast growth detection," Biosens. Bioelectron., vol. 21, pp. 849-856, 2005.

[34] B. Ilic, D. Czaplewski, H. G. Craighead, P. Neuzil, C. Campagnolo, and C. Batt, "Mechanical resonant immunospecific biological detector," Appl. Phys. Lett., vol. 77, pp. 450-452, 2000.

[35] A. P. Davila, J. Jang, A. K. Gupta, T. Walter, A. Aronson, and R. Bashir, "Microresonator mass sensors for detection of bacillus anthracis sterne spores in air and water," Biosens. Bioelectron., vol. 22, pp. 3028-3035, 2007.

[36] S. S. Verbridge, L. M. Bellan, J. M. Parpia, and H. G. Craighead, "Optically driven resonance of nanoscale flexural oscillators in liquid," Nano Lett., vol. 6, pp. 2109-2114, 2006.

[37] T. P. Burg and S. R. Manalis, "Suspended microchannel resonators for biomolecular detection," Appl. Phys. Lett., vol. 83, pp. 2698-2700, 2003. 
[38] M. Su, S. Li, and V. P. Dravid, "Microcantilever resonance-based DNA detection with nanoparticle probes," Appl. Phys. Lett., vol. 82, pp. 3562-3564, 2003.

[39] T. Kwon, K. Eom, J. Park, D. S. Yoon, H. L. Lee, and T. S. Kim, "Micromechanical observation of the kinetics of biomolecular interactions," Appl. Phys. Lett., vol. 93, pp. 173901, 2008.

[40] J. H. Lee, T. S. Kim, and K. H. Yoon, "Effect of mass and stress on resonant frequency shift of functionalized $\mathrm{Pb}(\mathrm{Zr} 0.52 \mathrm{Ti} 0.48) \mathrm{O}-3$ thin film microcantilever for the detection of C-reactive protein," Appl. Phys. Lett., vol. 84, pp. 3187-3189, 2004.

[41] M. Varshney, P. S. Waggoner, C. P. Tan, K. Aubin, R. A. Montagna, and H. G. Craighead, "Prion protein detection using nanomechanical resonator arrays and secondary mass labeling," Anal. Chem., vol. 80, pp. 2141-2148, 2008.

[42] T. Kodadek, "Biochemistry: molecular cloaking devices," Nature, vol. 453, pp. 861-862, 2008.

[43] J. S. Sebolt-Leopold and J. M. English, "Mechanisms of drug inhibition of signalling molecules," Nature, vol. 441, pp. 457-462, 2006.

[44] I. Kozinsky, H. W. C. Postma, I. Bargatin, and M. L. Roukes, "Tuning nonlinearity, dynamic range, and frequency of nanomechanical resonators," Appl. Phys. Lett., vol. 88, pp. 253101, 2006.

[45] H. W. C. Postma, I. Kozinsky, A. Husain, and M. L. Roukes, "Dynamic range of nanotube- and nanowire-based electromechanical systems," Appl. Phys. Lett., vol. 86, pp. 223105, 2005.

[46] X. M. H. Huang, X. L. Feng, C. A. Zorman, M. Mehregany, and M. L. Roukes, "VHF, UHF and microwave frequency nanomechanical resonators," New J. Phys., vol. 7, pp. 247-247, 2005.

[47] M. D. Dai, K. Eom, and C.-W. Kim, "Nanomechanical mass detection using nonlinear oscillations," Appl. Phys. Lett., vol. 95, pp. 203104, 2009.

[48] L. Meirovitch, Analytical methods in vibrations, Macmillan, New York, 1967.

[49] J. T. Oden, E. B. Becker, and G. F. Carey, Finite Elements: An Introduction(Vol. 1), Prentice Hall, 1981.

[50] J. T. Oden and J. N. Reddy, An Introduction to the Mathematical Theory of Finite Elements, John Wiley \& Sons, New York, 1976.

[51] J. K. Hale, Oscillations in Nonlinear Systems, McGraw-Hill, New York, 1963.

[52] C. Hayashi, Nonlinear Oscillations in Physical Systems, McGraw-Hill, New York, 1964.

[53] S. Cherian and T. Thundat, "Determination of adsorption-induced variation in the spring constant of a microcantilever," Appl. Phys. Lett., vol. 80, pp. 2219-2221, 2002.

[54] A. W. McFarland, M. A. Poggi, M. J. Doyle, L. A. Bottomley, and J. S. Colton, "Influence of surface stress on the resonance behavior of microcantilevers," Appl. Phys. Lett., vol. 87, pp. 053505, 2005.

[55] K. S. Hwang, K. Eom, J. H. Lee, D. W. Chun, B. H. Cha, D. S. Yoon, T. S. Kim, and J. H. Park, "Dominant surface stress driven by biomolecular interactions in the dynamical response of nanomechanical microcantilevers," Appl. Phys. Lett., vol. 89, pp. 173905, 2006.

[56] J. Dorignac, A. Kalinowski, S. Erramilli, and P. Mohanty, "Dynamical response of nanomechanical oscillators in immiscible viscous fluid for in vitro biomolecular recognition," Phys. Rev. Lett., vol. 96, pp. 186105, 2006.

[57] M. E. Gurtin, X. Markenscoff, and R. N. Thurston, "Effect of surface stress on the natural frequency of thin crystals," Appl. Phys. Lett., vol. 29, pp. 529530, 1976

[58] M. J. Lachut and J. E. Sader, "Effect of Surface Stress on the Stiffness of Cantilever Plates," Phys. Rev. Lett., vol. 99, pp. 206102, 2007.

[59] M. J. Lachut and J. E. Sader, "Effect of surface stress on the stiffness of cantilever plates: Influence of cantilever geometry," Appl. Phys. Lett., vol. 95, pp. 193505, 2009.

[60] M. F. Hagan, A. Majumdar, and A. K. Chakraborty, "Nanomechanical Forces Generated by Surface Grafted DNA," J. Phys. Chem. B, vol. 106, pp. 10163-10173, 2002.

[61] K. Eom, T. Y. Kwon, D. S. Yoon, H. L. Lee, and T. S. Kim, "Dynamical response of nanomechanical resonators to biomolecular interactions," Phys. Rev. B., vol. 76, pp. 113408, 2007.

[62] X. Yi and H. L. Duan, "Surface stress induced by interactions of adsorbates and its effect on deformation and frequency of microcantilever sensors," $J$. Mech. Phys. Solids, vol. 57, pp. 1254-1266, 2009.

[63] G.-Y. Huang, W. Gao, and S.-W. Yu, "Model for the adsorption-induced change in resonance frequency of a cantilever," Appl. Phys. Lett., vol. 89, pp. 043506, 2006

[64] E. Stern, R. Wagner, F.J. Sigworth, R. Breaker, T.M. Fahmy, and M.A. Reed, "Importance of the debye screening length on nanowire field effect transistor sensors," Nano Lett., vol. 7, pp. 3405-3409, 2007.

[65] Y. Cui, Q. Wei, H. Park, and C.M. Lieber, "Nanowire nanosensors for highly sensitive and selective detection of biological and chemical species," Sci- 
ence, vol. 293, pp. 1289-1292, 2001.

[66] M. Curreli, R. Zhang, F. N. Ishikawa, H.-K. Chang, R. J. Cote, C. Zhou, and M. E. Thompson, "Realtime, label-free detection of biological entities using nanowire-based fets," IEEE Trans. Nanotechnol., vol. 7, pp. 651-667, 2008.

[67] Z. Gao, A. Agarwal, A. D. Trigg, N. Singh, C. Fang, C.-H. Tung, Y. Fan, K. D. Buddharaju, and J. Kong, "Silicon nanowire arrays for label-free detection of DNA," Anal. Chem., vol. 79, pp. 3291-3297, 2007.

[68] K.-Y. Park, Y.-S. Sohn, C.-K. Kim, H.-S. Kim, Y.S. Bae, and S.-Y. Choi, "Developemt of FET-type albumin sensor for diagnosing nephritis," Biosens. Bioelectron., vol. 23, pp. 1904-1907, 2008.

[69] F. Patolsky, B. T. Timko, G. Zheng, and C. M. Lieber, "Nanowire-based nanoelectronic devices in the life sciences," MRS Bull., vol. 32, pp. 142-149, 2007.

[70] M. J. Schöning and A. Poghossian, "Recent advances in biologically sensitive field-effect transistors (BioFETs)," Analyst, vol. 127, pp. 1137-1151, 2002.

[71] A. Kim, C. S. Ah, H. Y. Yu, J.-H. Yang, I. B. Baek, C.-G. Ahn, C. W. park, M. S. Jun, and S. Lee, "Ultrasensitive, labe-free, and real-time immunodetection using silicon field-effect transistors," Appl. Phys. Lett., vol. 91, pp. 103901, 2007.

[72] W. U. Wang, C. Chen, K.-H. Lin, Y. Fang, and C. M. Lieber, "Label-free detection of small-moleculeprotein interactions by using nanowire nanosen- sors," Proc. Natl. Acad. Sci., vol. 102, pp. 32083212, 2005.

[73] F. Patolsky, G. Zheng, and C. M. Lieber, "Fabrication of silicon nanowire devices for ultrasensitive, label-free, real-time detection of biological and chemical species," Nat. Protoc., vol. 1, pp. 17111724, 2006.

[74] E. Stern, J. K. Klemic, D. A. Routenberg, P. N. Wyrembak, D. B. Turner-Evans, A. D. Hamilton, D. A. LaVan, T. M. Fahmy, and M. A. Reed, "Labelfree immunodetection with CMOS-compatible semiconducting nanowires," Nature Letters, vol. 445, pp. 519-522, 2007.

[75] Y. Chen, X. Wang, S. Erramilli, P. Mohanty, and A. Kalinowski, "Silicon-based nanoelectronic field-effect pH sensor with local gate control," Appl. Phys. Lett., vol. 89, pp. 223512, 2006.

[76] Y.-S. Sohn, G. B. Kang, and Y. T. Kim, "Fabrication of silicon nanowire field-effect transistor biosensor by CMOS compatible top-down approach with descum process," Sensor Lett., vol. 7, pp. 1039-1043, 2009.

[77] C. W. Park, C.-G. Ahn. J.-H. Yang, I.-B. Baek, C. S. Ah, A. Kim, T.-Y. Kim, and G. Y. Sung, "Control of channel doping concentration for enhancing the sensitivity of 'top-down' fabricated Si nanochannel FET biosensors," Nanotechnology, vol. 20, pp. 475501, 2009.

\section{엄 길 호}

- 센서학회지 제 19 권, 제 3 호, p. 220 , 참조

\section{권 태 윤}

- 센서학회지 제 19 권, 제 3 호, p. 220 , 참조

\section{손 영 수}

- 센서학회지 제 19 권, 제 2 호, p. 148 , 참조 